\title{
SOLID LIPID NANOPARTICLES (SLNS) AS NANO DRUG DELIVERY CARRIERS: PREPARATION, CHARACTERIZATION AND APPLICATION
}

\author{
MONA QUSHAWY1,3*, ALI NASR ${ }^{2,3}$ \\ ${ }^{1}$ Department of Pharmaceutics, Faculty of Pharmacy, University of Tabuk, Tabuk, Saudi Arabia, ${ }^{2}$ Department of Pharmaceutics, Faculty of \\ Pharmacy, Port Said University, Port Said, Egypt, ${ }^{3}$ Department of Pharmaceutics, Faculty of Pharmacy, Sinai University, Alarish, Egypt \\ Email: mqushawy@ut.edu.sa \\ Received: 18 Jul 2019, Revised and Accepted: 22 Oct 2019
}

\begin{abstract}
Over the last few years, there has been a significant consideration of solid lipid nanoparticles (SLNs) as an alternative method to other colloidal dispersion methods for drug delivery. Special consideration has been given to the use of SLNs as a drug carrier in recent years. SLNs are aqueous dispersions in which the colloidal particles consist of solid lipids that are biodegradable. As a result of their physical stability, the protection of the entrapped drug from decomposition, the provision of controlled drug release, and the exceptional acceptability, SLNs have several advantages over other drug carriers. This article focuses on the techniques of SLNs preparation and characterization, the effect of formulation variables on SLNs properties, the routes of administration, and the pharmaceutical applications. The data used for this review was collected by searching on Google Scholar and PubMed using the following keywords during the period from 2010 to date.
\end{abstract}

Keywords: Solid lipid nanoparticles, Colloidal dispersions, Pharmaceutical applications

(c) 2020 The Authors. Published by Innovare Academic Sciences Pvt Ltd. This is an open-access article under the CC BY license (http://creativecommons.org/licenses/by/4.0/) DOI: http://dx.doi.org/10.22159/ijap.2020v12i1.35312 Journal homepage: https://innovareacademics.in/journals/index.php/ijap

\section{INTRODUCTION}

Novel drug delivery systems are rapidly developing as a result of the success achieved in disciplines such as biomedical engineering, biotechnology, and nanotechnology. Several methods of preparation are used nanotechnology in the production of Nano-sized structures. In pharmacy, the aim of nanotechnology is to prepare drugs in the form of nanoparticles, which can be effectively absorbed, resulting in giving a pharmacological response with minimum side effects via a controlled drug release method [1]. As colloidal particles, SLNs are made up of solid lipids with a very high melting point forming a solid base covered by a hydrophilic surfactant. The use of solid lipid as a drug carrier is highly recognized by lipid pellets for oral drug transportation [2]. Lipids, which include glycerides, waxes, fat, oil, triglycerides, and hard fat, are used in the preparation of SLNs. They provide advantages for SLNs as a result of the lipid matrix consisting of physiological lipids with a low hazard of severe and chronic toxicity. It was reported that the solid lipid increases the controlled release properties and enhances the chemical stability of entrapped drugs. These advantages of solid lipid over liquid lipid can be attributed to the physicochemical properties associated with the physical condition of the lipid state [3]. Several studies have reported the use of a solid lipid in the preparation of SLNs instead of a liquid lipid, which allows the slow digestion of the nanoparticles resulting in the continued release of the entrapped drugs. Aqueous surfactants are other main excipients of SLNs. They act as an emulsifier to produce $\mathrm{o} / \mathrm{w}$ emulsion and as a stabilizer for the dispersion of SLNs. Their selection depends primarily on the route of application. Generally, SLNs are prepared by a solid lipid from which the drug is dissolved or diffused [4].

\section{The effect of formulation variables on the properties of SLNs}

For the construction of any drug transport scheme, excipients are vital and have the foremost part in defining product standards and efficiency [5].

\section{$>$ The effect of lipid}

The effect of lipid type on the properties of SLNs was observed by the hot homogenization method. A lipid with a high melting point was reported to form SLNs with a large size due to the higher viscosity of the diffused phase. Lipid hydrophobicity, lipid crystals forms, and lipid crystallization also represent factors which affect the SLNs' properties [6]. Lipids are mixes of diverse compounds, and for this reason, their production may differ from a batch to another.
In addition, the suppliers and such slight alterations of lipids can influence the quality of SLNs. Zeta potential, in vitro drug release, and particle size are the properties that are affected by the type of lipid. Chakraborty et al. reported that the increase in the lipid content over $5-10 \%$ would give rise to a larger particle size of SLNs [7].

\section{$>$ Effect of surfactant}

The properties of SLNs are greatly affected by the type of emulsifier. Any decrease in the surface tension and the particle size during preparation will be achieved when there is an increase in the quantity of emulsifier. Any decrease in the particle size will give rise to a large surface area. During the preparation of the primary dispersion of SLNs, an excess amount of surfactant should be added to cover the surfaces of the formed nanoparticles through HighPressure Homogenization [8]. The time required for the distribution of the surfactant between the surfaces of the prepared particles and micelles differs dependent on the type of surfactant. Previous studies revealed that the surfactants with low molecular weight take less time for redistribution, while surfactants with high molecular weight take a longer time to redistribute. When sodium glycocholate, a co-emulsifying agent, is added, it gives a further decrease in the size of the prepared nanoparticles [9].

\section{The principle of drug release from SLN}

Ramteke et al. listed the following as the standards of drug release from lipid nanoparticles [10]:

- A large surface area will result in a higher medication discharge due to little molecule measured in the nanometer.

- If the drug is evenly spread in the lipid matrix, a slow medication discharge is achieved.

- $\quad$ Fast medication release is obtained when the lipid carrier has a crystalline behaviour, and the drug has high portability.

- The fast-drug release in the initial five minutes in the drugenriched shell model is due to the high surface area of the drug particles which are deposited on the surface.

- $\quad$ The drug release decreases with the increase in the size of the particle, and sustained release can be attained when there are sufficient large particles (lipid macromolecules). 
- The type and amount of the used surfactant existing in the outer shell of the SLNs have a significant effect on drug release. The small amount of surfactant can give rise to minimal burst and prolonged drug discharge.

- The size of the particle and the drug release behavior are affected by several parameters such as SLN composition (drug, lipid, and surfactant), production technique, equipment, lyophilization, sterilization, and production time.

\section{SLN drug incorporation models}

The discharge of drug from SLN is explained through the following drug incorporation models (table 1 and fig. 1):

$>$ Homogenous matrix model or SLN Type I

$>$ Drug enhanced shell model or SLN Type II

$>$ Drug enhanced core model or SLN Type III

Table 1: Different drug incorporation models in SLN

\begin{tabular}{|c|c|c|c|}
\hline SLN type I & SLN type II & SLN type III & References \\
\hline $\begin{array}{l}\text { Cold homogenization techniques are } \\
\text { used to formulate this type }\end{array}$ & $\begin{array}{l}\text { Hot homogenization technique is used to } \\
\text { formulate this type }\end{array}$ & $\begin{array}{l}\text { Cold dispersion allows super-saturation of } \\
\text { the active ingredient and hence dissolving } \\
\text { the drug in the lipid }\end{array}$ & 11 \\
\hline No solubilizing agent is used & $\begin{array}{l}\text { The lipid core is prepared at the } \\
\text { recrystallization temperature of the lipid }\end{array}$ & The precipitation of drug is melted lipid & 12 \\
\hline $\begin{array}{l}\text { The drug is dispersed in the lipid } \\
\text { matrix }\end{array}$ & $\begin{array}{l}\text { Cooling the obtained dispersion results in } \\
\text { partitioning the drug into the lipid phase }\end{array}$ & $\begin{array}{l}\text { Finally, the recrystallization of the lipid is } \\
\text { done by further cooling }\end{array}$ & 13 \\
\hline $\begin{array}{l}\text { There is a strong interaction between } \\
\text { drug and lipid }\end{array}$ & The drug is concentrated in the outer shell & The drug is concentrated in the core & 14 \\
\hline
\end{tabular}

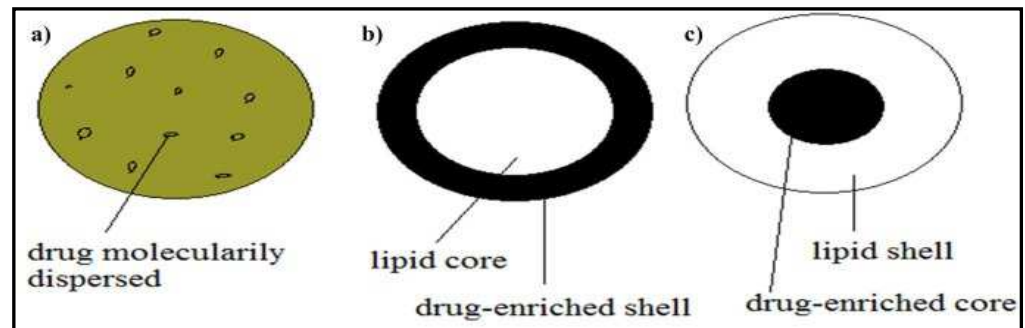

Fig. 1: Diagrammatic illustration of drug integration models of SLNs [11]

\section{$>$ Homogenous matrix model}

Type I is generated from a solid solution of lipid and active constituent. The solid solution is obtained when SLNs are prepared by a cold homogenization technique without using a surfactant. A lipid mixture can be made with the active constituent in a molecularly dispersed form. Upon solidifying the lipid mixture, it is crushed in its solid form to reduce the accumulation of the drug in various parts of SLNs [12].

\section{$>\quad$ Drug enriched shell model}

SLN type II is produced using the hot high-pressure homogenization technique and applying a small concentration of the active ingredient in the lipid matrix. In the process of cooling the hot $0 / \mathrm{W}$ nano-emulsion, the lipid is precipitated first. As a result, there is an increase in the concentration of the drug molecules, which remained in the melt [13]. Then; the outer shell is solidified containing both lipid and drug. The active constituent percentage contained in the outermost shell can be altered in a regulated shell design when the coenzyme is incorporated [14].

\section{$>\quad$ Drug enriched core model}

When the concentration of the active ingredient in the lipid melt is high and comparatively near saturation, the core model is developed. In this method, cooling Nano-emulsion leads to the precipitation of the drug due to super saturation at low temperatures. Further cooling leads to the precipitation of the melted lipid and surrounding the precipitated drug particles and the development of drug enriched core [14].

\section{Advantages and disadvantages of SLNs as drug carrier}

\section{Advantages of SLNs}

- $\quad$ The use of biodegradable lipids reduces the possibilities of severe and prolonged toxicity.
- Enhancing the bioavailability of low water-soluble active constituents.

- Enhancing the stability of chemically labile drugs through protection from the external environment.

- $\quad$ SLNs have improved stability in comparison with other drug carriers as liposomes.

- The high entrapment efficiency of the active constituents.

- The possibility of lyophilisation.

Disadvantages of SLN

- $\quad$ The drug loading ability is poor.

- Water content in the dispersions is comparatively high (70-99.9\%).

- The unpredictable tendency to gelation.

- The unpredicted dynamics of polymeric changes.

- Drug expulsion during storage after a polymeric transition.

- The possibility of particle growth.

\section{Techniques for SLNs preparation}

The properties of SLNs are greatly influenced by the techniques used in their production. Among these properties are the following: the size of the particle, the drug loading ability, the drug release behavior, and the drug stability. Due to these effects, the efficacy of their preparation also depends on the method of preparation. Several methods are used for manufacturing finely distributed SLNs [15].

\section{$>\quad$ High shear homogenization (HSH)}

This method is a potent approach to SLNs preparation. For the large scale production of SLNs, HSH can be employed in contrast to other 
methods. The hot and cold methods are the two methods of homogenization. In both methods, before the shear homogenization, the active constituents are melted or spread in the melted lipid. Nano-sized SLNs are obtained when the pressure of 100-2000 bar in the homogenizer causes the fluid to move in the narrow gap. The improved drug stability, Large-scale production, improved drug loading, and the lack of organic solvent are the advantages of homogenization $[16,17]$.

\section{$>$ Hot homogenization}

In this process, the homogenization process takes place at a temperature greater than the melting point of the lipid. The Preemulsions are formed when drug-loaded lipid melt is dispersed in hot aqueous solution of surfactants using Ultra-Turrax (mixing device). As a result of the decreased viscosity due to high temperatures, the size of the particle becomes smaller (fig. 2). Three basic problems were reported for this method as follows: the difficulty of using thermolabile drug, the penetration of the drug into the aqueous phase during the homogenization process, and the complexity of the crystallization step of the Nano-emulsion, which may result in modification and/or super-cooled melts [18]. Remya and Damodharan prepared cilnidipine-loaded SLNs using the hot homogenization method [19].

\section{$>$ Cold homogenization}

In this approach, the drug is melted in the lipid melt, and after that, it is quickly cooled using liquid nitrogen or ice crystal. Milling leads to the production of nanoparticles in the range of 50-100 nm, which can be dispersed in a cold surfactant that produces a pre-suspension. Highpressure homogenization (PHP) is used to split the nanoparticles into SLNs at the ambient temperature. All the main drawbacks of the hot homogenization approach are resolved by the cold homogenization procedure (fig. 2) [20]. Karami et al. prepared Superoxide dismutaseloaded SLNs using the cold homogenization method [21].

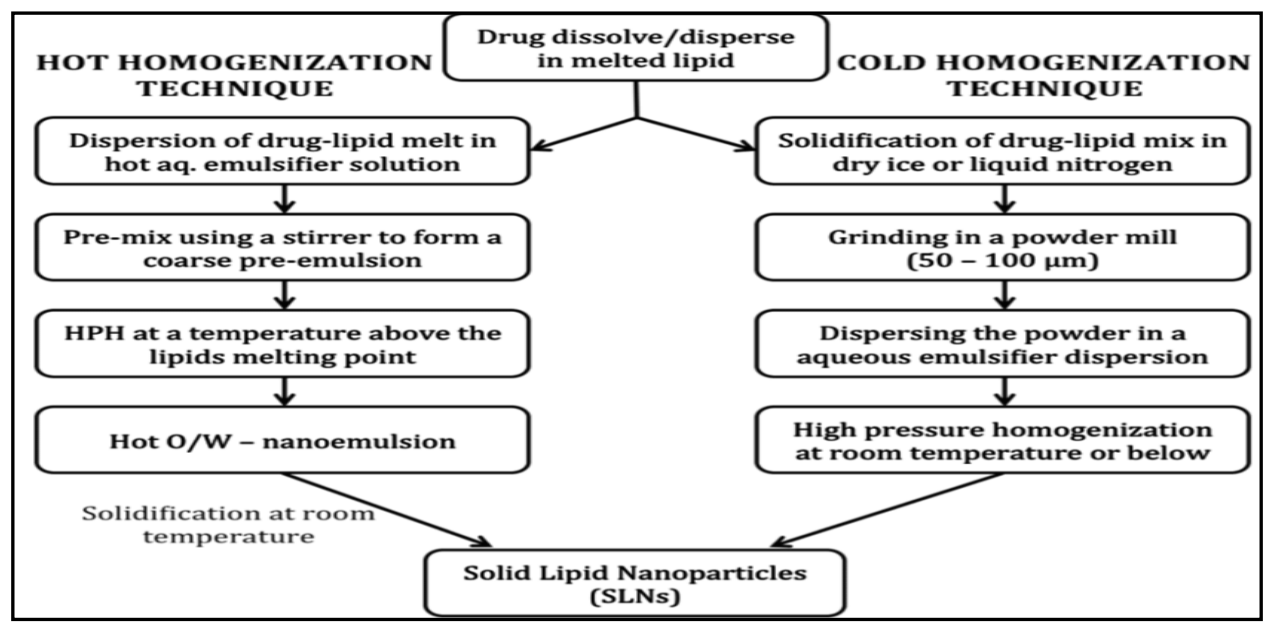

Fig. 2: Cold and hot homogenization procedures [22]

\section{$>$ Ultra-sonication or high-speed homogenization}

This technique is one of the techniques which are used to produce SLNs. In this technique, the aqueous phase containing a large quantity of surfactant is dispersed with the lipid phase, as shown in fig. 3. The large quantity of the surfactant is considered as a drawback. The inability to produce nanoparticles with a narrow size distribution, thereby giving rise to instability during storage, is also considered as a drawback for this technique. This method, unlike the hot and cold homogenization, makes use of simple instruments that are readily available almost in all laboratories [23, 24]. Swidan et al. prepared SLNs of Paclitaxel using this method [25]. Shelat et al. prepared curcuminoid-loaded SLNs to improve the oral bioavailability using the high-speed homogenization method [26].

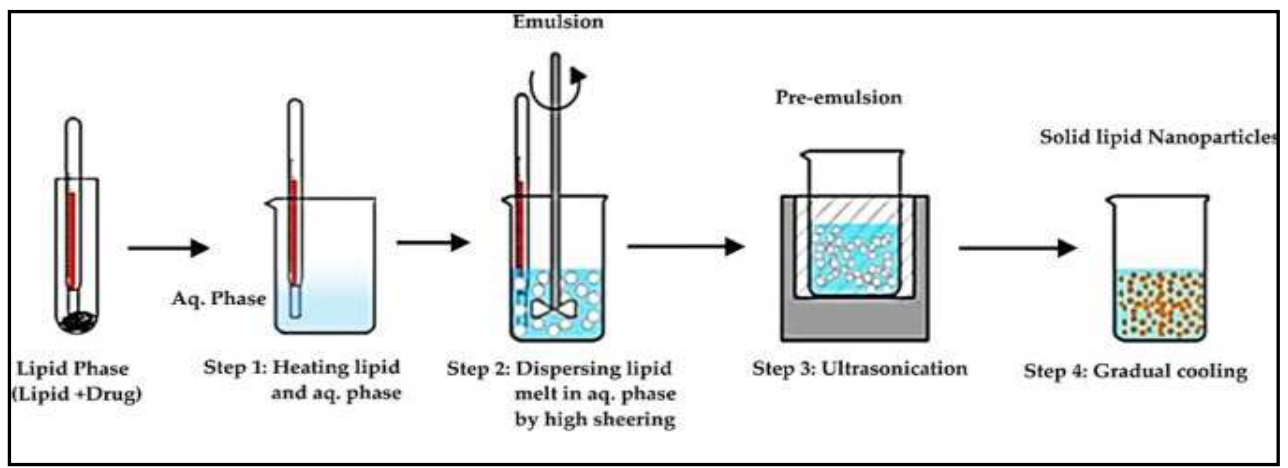

Fig. 3: Ultra sonication technique [27]

\section{$>$ Spray drying}

This method employs lipids with a high melting point $\left(\geq 70^{\circ} \mathrm{C}\right)$. It is an alternative to the lyophilization method (fig. 4). A better result is obtained when a $1 \%$ concentration of SLN is used in a suspension of trehalose in water, or $20 \%$ trehalose is used in the ethanol-water mixture [18].

Wang et al. prepared ultra-fine powders of polysaccharide-coated SLN carriers using the innovative Nano-spray drying technique [28]. 


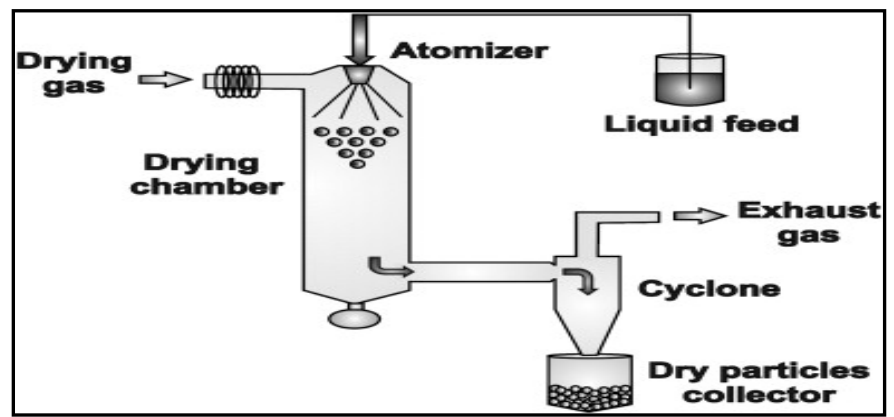

Fig. 4: Spray drying technique [29]

\section{$>$ Solvent emulsification-diffusion method}

As shown in fig. 5, in this technique, a water-immiscible organic solvent is employed to dissolve the solid lipid. The emulsion is formed by dispersing the lipid phase in an aqueous phase containing a surfactant. The organic solvent is allowed to evaporate from the emulsion at reduced pressure. As a result of organic solvent evaporation, the SLNs are prepared in the aqueous phase (by applying the lipid precipitation process in the aqueous phase). This process does not undergo thermal stress; however, the use of an organic solvent is considered as a disadvantage. The size of the particle differs according to the solid lipid and surfactant $[30,31]$. Patravale and Mirani prepared SLNs-based gel for topical use using the solvent emulsification-diffusion method [32].

\section{$>$ Solvent evaporation method}

Cyclohexane, which is a water-immiscible organic solvent, is used to dissolve the solid lipid, and then it is emulsified with the aqueous phase. When the solvent is evaporated, nanoparticles dispersal is obtained by the deposition of the lipid in the aqueous phase by providing the nanoparticles of $25 \mathrm{~nm}$ mean size. By using highpressure homogenization, the lipid solution is emulsified in the aqueous phase (fig. 5). Under the reduced pressure of 40-60 $\mathrm{m}$ bar, cyclohexane was removed from the emulsion by evaporation [33]. Amasya et al. prepared 5-fluorouracil-loaded SLNs using the solvent evaporation method [34].

\section{$>$ Solvent-injection method}

In this method, the active ingredient and the lipid are dissolved in a water-soluble organic solvent such as isopropanol and ethanol. The solution is injected into water under stirring using a syringe needle. The lipid is converted into nanoparticles when it becomes in contact with water, resulting in entrapping the drug. The lipid type, the solvent applied, the surfactant, and the viscosity of the external phase are factors that determine the size of the particle (fig. 6). Leonardi et al. designed and prepared Ciprofloxacin Hydrochloride as SLNs using the solvent-injection method [36].

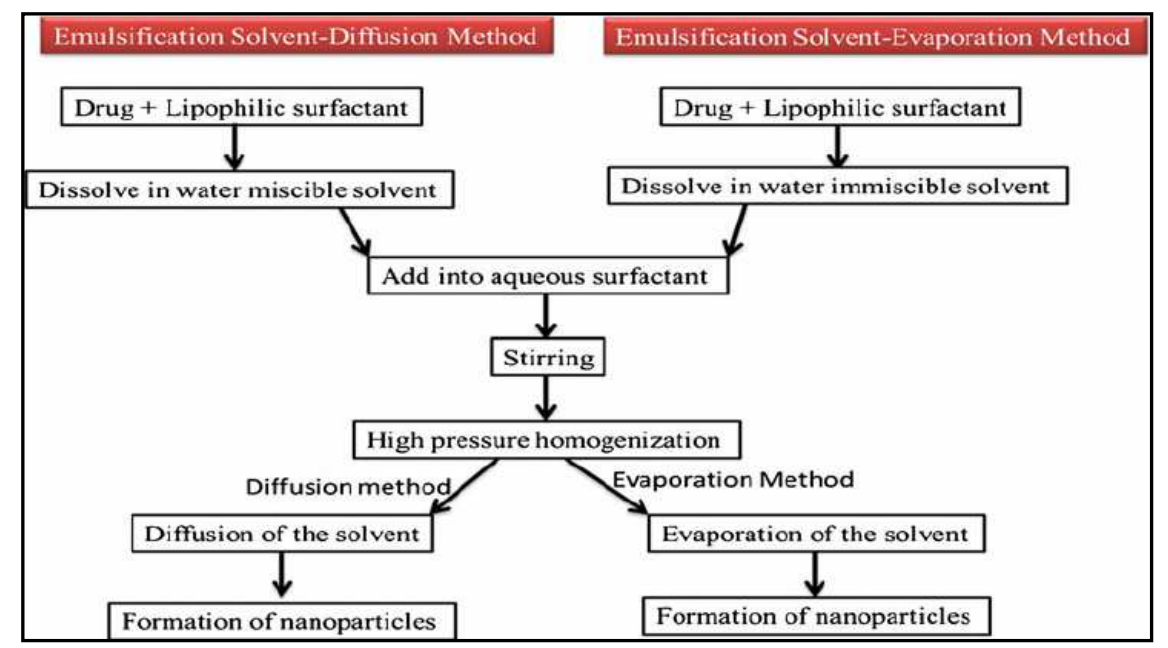

Fig. 5: Solvent emulsification-diffusion and evaporation technique [35]

\section{$>$ Double emulsification method}

This method is derived from the emulsion solvent evaporation technique. This method is employed to produce SLNs loaded with hydrophilic drugs. In this method, the drug and emulsifying agents are existing in the aqueous internal phase of $\mathrm{w} / \mathrm{o} / \mathrm{w}$ double emulsion [38].

\section{$>$ Supercritical fluid extraction of emulsions (SFEE)}

Comparatively, this technique is novel for the preparation of SLNs. This technique uses carbon dioxide (a supercritical fluid) for removing the solvent from o/w emulsions (fig. 7). $\mathrm{CO}_{2}$ is a better choice; however, it cannot dissolve many drugs. Hence, the supercritical anti-solvent precipitation (SAS) can serve as a substitute for SFEE [39]. Couto et al. prepared Vitamin $B_{2}$ in SLNs using supercritical CO2 [40].

\section{$>$ Membrane contractor method}

This method uses a membrane contractor for large scale production of SLN. In this method, the lipid phase is allowed to press through the membrane pores at a temperature above the lipid's melting point. As a result, the lipid is extruded in the form of melted small droplets through the membrane pores. The aqueous phase circulates inside the membrane module and sweeps away the lipid droplets, which are formed at the pores outlet.

The effect of process parameters (such as aqueous phase flow velocity, lipid phase pressure, aqueous phase, and lipid phase temperature, and size of the membrane pore) on the size of SLNs and the lipid phase extrusion is studied [41]. Also, the stability of vitamin-E-laden SLN was achieved (fig. 8). 


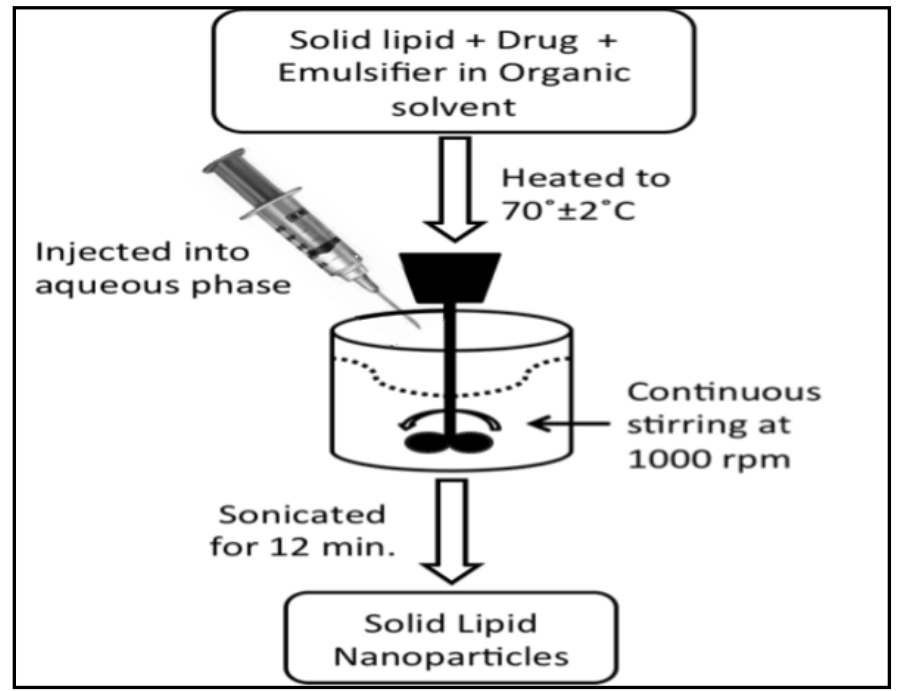

Fig. 6: Solvent-injection method [37]

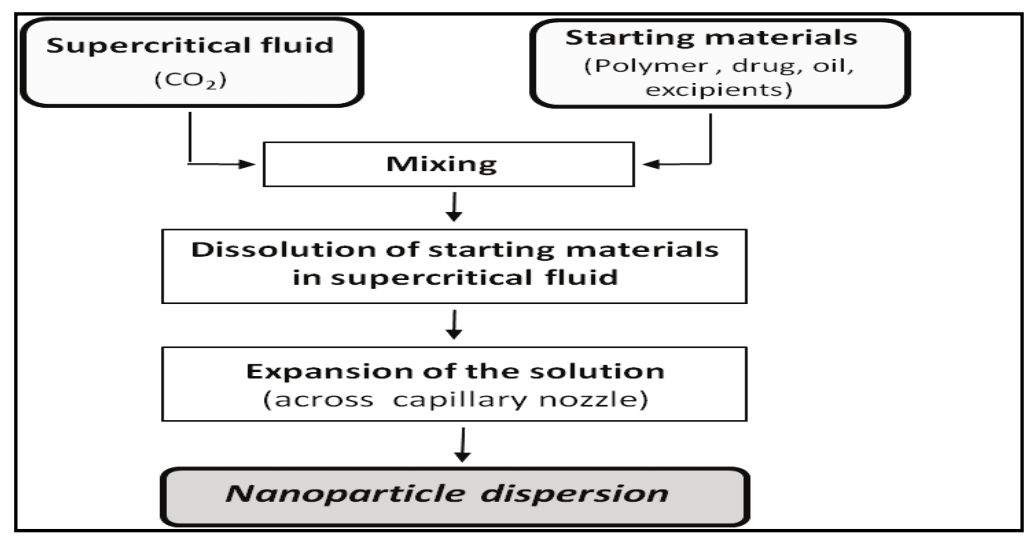

Fig. 7: Supercritical fluid technology technique [37]

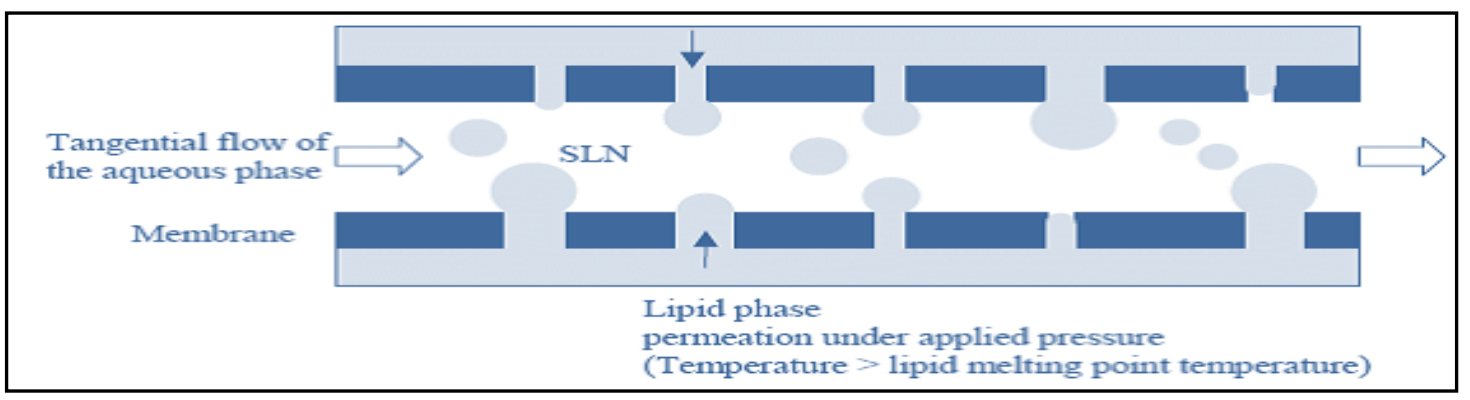

Fig. 8: Membrane contractor for SLN preparation [1]

\section{Secondary production methods}

\section{$>$ Freeze-drying}

In this method, water is removed to enhance the physicochemical stability of the system. In the pharmaceutical industry, freezedrying is the most frequently used method for converting solutions or suspensions into solids of high stability. Freeze-drying is also called lyophilization in which the frozen water is removed by sublimation.

\section{$>$ Sterilization}

The SLNs dispersion, which is intended for parenteral administration, should be sterile. To achieve sterilization, filtration, autoclaving, aseptic production, and gamma irradiation are generally used for the production of sterile SLNs. The sterilization using autoclave is the most common and suitable process. However, the autoclaving is an excellent method for sterilization, where the elevated temperatures during sterilization can give rise to the aggregation of the SLNs [42].

\section{Characterization of SLNs}

The use of advanced microscopic methods including Transmission Electron Microscopy (TEM), Scanning Electron Microscopy (SEM) and Atomic Force Microscopy (AFM) has made it possible to characterize nanoparticles according to their morphology, surface charge, and size. In vivo distribution, the nanoparticles and physical stability are influenced by the distribution of their size and the mean particle diameter and charge [12]. 


\section{$>$ Particle size analysis and Zeta potential}

The physical stability of SLNs is determined by the size of the particle. The most common techniques for particle size determination are the Laser Diffraction (LD) and Photon Correlation Spectroscopy (PCS). The PCS, also called dynamic light scattering, is employed to determine the intensity of the scattered light resulting from the random motion of the particles. The PCS is employed to determine particle size within the range of $3 \mathrm{~nm}$ to $3 \mu \mathrm{m}$ and laser diffraction is used to determine the particle size for the range of 100 $\mathrm{nm}$ to $180 \mu \mathrm{m}$. Although the PCS is a good instrument for nanoparticle characterization, it can also be used for larger size determination of the micro-particles. The LD technique is based on the effect of the particle size on the diffraction. Smaller particles, compared to the large ones, produce stronger scattering with high diffraction angle [12].

Zeta meter or Zeta potential analyzer can be used for the determination of Zeta potential. For size determination and zeta potential determination, the dispersion of SLNs is first diluted 50fold with the primary dispersion preparation medium [43]. The increased value of zeta potential could give rise to the disaggregation of the particles. Zeta potential determination makes it possible to predict the storage stability of colloidal dispersion [44].

\section{$>$ X-ray diffraction and differential scanning calorimeter (DSC)}

The geometric scattering of radiation from crystal planes within a solid allows the determination of the presence or absence of the former and thus permits the measurement of the degree of crystallinity. DSC can be applied to evaluate the properties and the degree of crystallinity of drugs in nanoparticles [45].

\section{$>$ Electron microscopy}

For direct observation of nanoparticles, TEM, and SEM are employed [46]. The SEM is used for better morphological examination, and it has a small size limit of detection [12].

\section{$>$ Atomic force microscopy (AFM)}

By using this approach, a probe tip with atomic-scale sharpness is re-established across a sample to create a topological map, which is based on the forces between the tip and the surface. The atomic force microscopy is a useful tool to obtain ultrahigh-resolution of the particles [45].

\section{$>$ Dynamic light scattering (DLS)}

The most common and the fastest methods for determining the particle size are the DLS and PCS. The DLS is commonly used for the size determination of Brownian nanoparticles in colloidal dispersion in the Nano and submicron range. Shining monochromatic light (laser) onto a solution of spherical particles (in case of random Brownian motion) leads to Doppler shift when the light hits the moving particles. As a result, the wavelength of the coming light is changed and it was revealed that this change is related to the size of the particle. The DLS is also useful for the determination of size distribution, the particle movement in the medium, the diffusion coefficient of the particle, and the use of the autocorrelation function. The PCS is the commonly applied method for the accurate determination of particle size and the distribution of size based on DLS [47].

\section{$>$ Nuclear magnetic resonance (NMR)}

NMR can be used for both the qualitative nature and the size of nanoparticles' measurement. The selection of the technique depends on the chemical shifting balances and the sensitivity to molecular flexibility to provide data about the physicochemical state of the constituent inside the nanoparticles [10].

\section{$>$ Acoustic methods}

Acoustic Spectroscopy is a technique that determines the particle size by measuring the attenuation of sound waves and applying the physical equation. The oscillating electric field created by the charged particle movement under the control of acoustic energy can be identified to provide information on the surface charge.

\section{$>$ Surface charge}

The interaction of Nanoparticles with their biological environment and their electrostatic interaction with bioactive compounds are determined by the intensity and nature of the surface charge. The zeta potential is used to analyze the colloidal stability of the nanoparticles. The zeta potential is an indirect measurement of the surface charge. The determination of zeta potential makes it possible to predict the stability of the colloidal dispersion during storage. The high value of zeta potential, whether positive or negative values, ensures the stability of the colloidal particle and also ensures the absence of aggregations (table 2). The degree of surface hydrophobicity then can be estimated from the values of the zeta potential. The zeta potential can also give information on the nature of the drug to be encapsulated within the nanocapsule or absorbed on the surface [48].

Table 2: Characterization parameters of SLNs

\begin{tabular}{lllll}
\hline S. No. & Parameters & Importance & Methods & References \\
\hline 1 & Size and Shape & Determining skin penetration & $\begin{array}{l}\text { Photon correlation, Scanning electron microscope } \\
\text { and Transmission electron microscope }\end{array}$ & 46 \\
& & Zeta potentiometer, Laser droplet anemometry & 48 \\
2 & Zeta potential & The stability of particles & Ultracentrifugation & 12 \\
3 & $\begin{array}{l}\text { Entrapment } \\
\text { efficiency }\end{array}$ & Suitability of method & & 6 \\
4 & $\begin{array}{l}\text { Drug content } \\
5\end{array}$ & The suitability of the method of preparation & UV, HPLC & 9 \\
\hline
\end{tabular}

\section{Routes of administration of SLNs}

\section{$>$ Perioral administration forms of SLN}

The oral SLNs may be administered in the form of an aqueous dispersion or SLNs loaded in the traditional forms of solid dosage like tablets, capsules, or pellets. The improved bioavailability and extended plasma levels were reported after the perioral administration of cyclosporine containing lipid Nano dispersions to animals. Kaliamurthi and Selvaraj found that SLNs are good carriers for oral administration of anti-diabetic drugs [49]. Ansari et al. supported the oral bioavailability of insulin by formulating it as SLNs [50]. Mahajan et al. prepared stearic-acid-based SLNs of candesartan cilextil to improve the oral bioavailability [51].

\section{$>$ Parenteral use}

Animals were injected intravenously with SLNs. The pharmacokinetic study of doxorubicin entrapped in SLN revealed the increased blood levels of the drug compared to the commercial drug suspension after intravenous administration in rats.

About the body distribution, SLN was reported to increase drug concentrations in the lung, spleen, and brain, while the traditional form of drug leads to the distribution of the drug into the liver and kidneys. Also, the injection of the drug in the form of SLNs reduces the irritant effect caused in the case of micro particles. Chetoni et al. prepared tobramycin SLNs for intraocular delivery [52]. 


\section{$>$ Transdermal application}

The dispersion of SLNs, which is prepared with low lipid content up to $5 \%$, was reported to have a very small particle size. The direct application of the dispersion of SLNs to the skin is difficult due to the low lipid concentration and low viscosity of the dispersion. To facilitate the application of SLNs to the skin, it is preferred to be incorporated into the base of cream, ointment, or gel. For direct application of the dispersion of SLNs, the concentration of lipids should be increased to produce a semisolid system suitable for the application to the skin [53]. Gönüllü et al. prepared SLNs of lornoxicam for transdermal delivery [54].

\section{$>$ Topical administration}

SLNs are very attractive colloidal carrier systems for skin applications due to the numerous desirable effects on the skin. Since they are made up of non-irritant and nontoxic lipids, this makes them appropriate for use on damaged or irritated skin. Jain et al. designed SLNs for the topical delivery of an anti-fungal drug [55].

\section{$>$ Ophthalmic administration}

The preparation of ophthalmic drugs as SLNs increases their efficacy due to the mucoadhesive characteristics of SLNs, which increase the ocular retention time and hence increase the bioavailability. Gowda et al. studied the effect of SLNs on enhancing the ocular availability of ophthalmic drugs [56]

\section{$>$ Respiratory application}

The nebulization of SLNs carrying anti-tubercular drugs, antiasthmatic drugs, and anti-cancer was found to be effective in increasing drug bioavailability and decreasing the dosing rate for improving the pulmonary action. Rosière et al. prepared paclitaxelloaded SLNs with improved efficacy in the treatment of lung tumors when used as inhalers [57].

\section{Pharmaceutical applications of SLNs}

SLNs have numerous pharmaceutical applications which include:

\section{$>$ Cancer treatment}

In recent years, various anticancer agents were compressed to SLNs and their in vitro, and in vivo efficiency were estimated.

\section{A) Targeting of anticancer drugs}

The extended-release of tamoxifen (an anticancer drug) is attained by preparing it as SLNs for intravenous administration in breast cancer. Also, the target effect of methotrexate and camptothecin to tumor tissue was accomplished with SLN loaded with the drug. Tupal et al. prepared doxorubicin-loaded SLNs for the treatment of skin cancer [58].

\section{B) SLN in breast cancer and lymph node metastases}

Mitoxantrone SLN local injections were produced to decrease the toxicity and increase the safety and bioavailability of the drug. The efficacy of doxorubicin in the reduction of breast cancer cells was reported to be enhanced when prepared as SLNs [59]. Wang et al. studied the anticancer effects of resveratrol-loaded SLNs on human breast cancer cells [60].

\section{$>$ Antitubercular chemotherapy}

The preparation of anti-tubercular drugs such as rifampicin and isoniazid as SLNs leads to the reduction of the dosage regimen and the improvement of patient compliance [61]. Bhandari et al. succeeded in preparing isoniazid-SLNs to improve bioavailability and prolong the therapeutic effect and therefore reduce pulsatile plasma concentrations [62].

\section{$>$ SLN as a carrier for vaccines}

Adjuvants are used to improve immune response during vaccination. The safer novel subunit vaccines are less efficient in immunization, and for this reason, efficient adjuvants are needed. Emulsion systems of SLNs have been recently employed to use the adjuvant. This is $\mathrm{O} / \mathrm{W}$ emulsions that are degraded in the body after administration [63].

\section{$>$ SLN for topical application}

SLNs are suitable colloidal transport systems for skin applications due to their numerous desirable effects on the skin. Recently, studies were carried out on SLNs with compounds such as ascorbyl palmitate, Vitamin E, retinol, clotrimazole, triptolide, nonsteroidal antiandrogen, tocopherol acetate and podophyllotoxin for topical application [64]. Kelidari et al. prepared spironolactone loaded SLNs for skin application [65].

\section{$>$ SLN for potential agriculture application}

Based on the previous study, it was found that when the volatile oil extracted from Artemisia arborescent L is incorporated into SLNS, it decreases the rapid evaporation compared to its incorporation into emulsions. This system is used in agriculture as an appropriate transporter of ecologically safe pesticides [12].

\section{$>$ Stealth nanoparticles}

This is a new system for drug-transport. It avoids the rapid clearance of the drug by the immune system. By using antibodylabeled stealth lipobodies, previous researches approved the improved delivery to the unreachable sites of target tissue [66].

\section{$>$ SLNs for cosmetics}

SLNs have been used in the production of sunscreens and they act as an important carrier for molecular sunscreen and ultraviolet (UV) blocker. The in vivo study revealed that skin moisturization would be improved by $31 \%$ after four weeks when $4 \%$ of SLN is added to the conventional cream. SLNs have shown to be a controlled, innovative, occlusive, and topical release [67]. Wissing and Müller found that the efficacy of hydrating cream and sunscreen creams improved when prepared in the form of SLNs [68].

\section{CONCLUSION}

SLNs are attractive drug carriers and are considered as an alternative method for other colloidal dispersion systems. The SLNs represent a safe and effective carrier for the drug due to the biocompatibility of the used solid lipid. This article covered the different methods of preparation of SLNs, the effect of formulation variable on the characteristics of SLNs, the advantages and disadvantages of SLNs, the characterization of SLNs, the route of administration, and the pharmaceutical applications.

\section{ACKNOWLEDGMENT}

The authors would like to acknowledge the help of the Research Consulting Unit, Faculty of Pharmacy, University of Tabuk, in checking the plagiarism for this manuscript.

\section{AUTHORS CONTRIBUTIONS}

All authors contributed equally to this work.

\section{CONFLICT OF INTERESTS}

The authors disclose that no conflicting interests associated with the manuscript exist.

\section{REFERENCES}

1. Yadav N, Khatak S, Sara U. Solid lipid nanoparticles-a review. Int J Appl Pharm 2013;5:8-18.

2. Muller R, Radtke M, Wissing S. Solid lipid nanoparticles (SLN) and nanostructured lipid carriers (NLC) in cosmetic and dermatological preparations. Adv Drug Delivery Rev 2002;54:131-55.

3. Mandawgade S, Patravale V. Development of SLNs from natural lipids: application to topical delivery of tretinoin. Int J Pharm 2008;363:132-8.

4. Amoabediny G, Haghiralsadat F, Naderinezhad S, Helder M, Akhoundi Kharanaghi E, Mohammadnejad Arough J, et al. Overview of preparation methods of polymeric and lipid-based (niosome, solid lipid, liposome) nanoparticles: a comprehensive review. Int J Polym Mater Polym Biomater 2018;67:383-400. 
5. Kakkar Thukral D, Dumoga SK, Mishra A. Solid lipid nanoparticles: promising therapeutic nanocarriers for drug delivery. Curr Drug Delivery 2014;11:771-91.

6. Mukherjee S, Ray S, Thakur RS. Solid lipid nanoparticles: a modern formulation approach in drug delivery system. Indian J Pharm Sci 2009;71:349.

7. Chakraborty S, Shukla D, Mishra B, Singh S. Lipid-an emerging platform for oral delivery of drugs with poor bioavailability. Eur J Pharm Biopharm 2009;73:1-15.

8. Weber S, Zimmer A, Pardeike J. Solid lipid nanoparticles (SLN) and nanostructured lipid carriers (NLC) for pulmonary application: a review of the state of the art. Eur J Pharm Biopharm 2014;86:7-22.

9. Mehnert W, Mäder K. Solid lipid nanoparticles: production, characterization, and applications. Adv Drug Delivery Rev 2012;64:83-101.

10. Ramteke K, Joshi S, Dhole S. Solid lipid nanoparticle: a review. IOSR J Pharm 2012;2:34-44.

11. Naseri N, Valizadeh H, Zakeri Milani P. Solid lipid nanoparticles and nanostructured lipid carriers: structure, preparation and application. Adv Pharm Bull 2015;5:305-13.

12. Garud A, Singh D, Garud N. Solid lipid nanoparticles (SLN): method, characterization, and applications. Int Cur Pharm J 2012;1:384-93.

13. Shirodkar RK, Kumar L, Mutalik S, Lewis S. Solid lipid nanoparticles and nanostructured lipid carriers: emerging lipid-based drug delivery systems. Pharm Chem J 2019;53:1-4.

14. Souto E, Muller R. Lipid nanoparticles (solid lipid nanoparticles and nanostructured lipid carriers) for cosmetic, dermal, and transdermal applications. Drugs Pharm Sci 2007;166:213.

15. Trotta M, Debernardi F, Caputo O. Preparation of solid lipid nanoparticles by a solvent emulsification-diffusion technique. Int J Pharm 2003;257:153-60.

16. Al Haj N, Abdullah R, Ibrahim S, Bustamam A. Tamoxifen drug loading solid lipid nanoparticles prepared by hot high-pressure homogenization techniques. Am J Pharmacol Toxicol 2008;3:219.

17. Ekambaram P, Sathali A, Priyanka K. Solid lipid nanoparticles: a review. Sci Rev Chem Commun 2012;2:80-102.

18. Muller RH, Mader K, Gohla S. Solid lipid nanoparticles (SLN) for controlled drug delivery-a review of state of the art. Eur J Pharm Biopharm 2000;50:161-77.

19. Pn Remya, Damodharan N. Formulation, development, and characterization of cilnidipine loaded solid lipid nanoparticles. Asian J Pharm Clin Res 2018;11:120-5.

20. Parhi R, Suresh P. Production of solid lipid nanoparticles-drug loading and release mechanism.J Chem Pharm Res 2010;2:211-27.

21. Karami MA, Zadeh BSM, Koochak M, Moghimipur E. Superoxide dismutase-loaded solid lipid nanoparticles prepared by cold homogenization method: characterization and permeation study through burned rat skin. Jundishapur J Nat Pharm Prod 2016;11:e33968.

22. Teja VC, Chowdary VH, Raju YP, Surendra N, Vardhan RV, Reddy BKK. A glimpse on solid lipid nanoparticles as drug delivery systems. J Global Trends Pharm Sci 2014;5:1649-57.

23. Manjunath K, Reddy J, Venkateswarlu V. Solid lipid nanoparticles as drug delivery systems. Methods Find Exp Clin Pharmacol 2005;27:127-44.

24. Patwekar S, Gattani S, Giri R, Bade A, Sangewar B, Raut V. Review on nanoparticles used in cosmetics and dermal products. World J Pharm Pharm Sci 2014;3:1407-21.

25. Swidan S, Ghonaim H, Samy A, Ghorab M. Comparative study of solid lipid nanoparticles and nanostructured lipid carriers for in vitro paclitaxel delivery. J Chem Pharm Res 2016;8:482-93.

26. Shelat P, Mandowara VK, Gupta DG, Patel S. Formulation of curcuminoid loaded solid lipid nanoparticles in order to improve oral bioavailability. Int J Pharm Pharm Sci 2015;7:278-82.

27. Ganesan P, Narayanasamy D. Lipid nanoparticles: different preparation techniques, characterization, hurdles, and strategies for the production of solid lipid nanoparticles and nanostructured lipid carriers for oral drug delivery. Sustainable Chem Pharm 2017;6:37-56.
28. Wang T, Hu Q, Zhou M, Xue J, Luo Y. Preparation of ultra-fine powders from polysaccharide-coated solid lipid nanoparticles and nanostructured lipid carriers by innovative nano spray drying technology. Int J Pharm 2016;511:219-22.

29. Sosnik A, Seremeta KP. Advantages and challenges of the spraydrying technology for the production of pure drug particles and drug-loaded polymeric carriers. Adv Colloid Interface Sci 2015;223:40-54.

30. Kamboj S, Bala S, Nair A. Solid lipid nanoparticles: an effective lipid-based technology for poorly water-soluble drugs. Int J Pharm Sci Rev Res 2010;5:78-90.

31. Mendoza Munoz N, Alcala Alcala S, Quintanar Guerrero D. Preparation of polymer nanoparticles by the emulsificationsolvent evaporation method: from Vanderhoff's pioneer approach to recent adaptations. In: Polymer Nanoparticles for Nanomedicines. Springer, Cham; 2016. p. 87-121.

32. Patravale VB, Mirani AG. Preparation and characterization of solid lipid nanoparticles-based gel for topical delivery. In: Pharmaceutical Nanotechnology, Humana, New York, NY; 2019. p. 293-302.

33. Kaur T, Slavcev R. Solid lipid nanoparticles: tuneable anticancer gene/drug delivery systems. In: Novel Gene Therapy Approaches. Intech Open; 2013.

34. Amasya G, Badilli U, Aksu B, Tarimci N. Quality by design case study 1: Design of 5-fluorouracil loaded lipid nanoparticles by the $\mathrm{W} / \mathrm{O} / \mathrm{W}$ double emulsion-solvent evaporation method. Eur J Pharm Sci 2016;84:92-102.

35. Kumar S, Dilbaghi N, Saharan R, Bhanjana G. Nanotechnology as emerging tool for enhancing the solubility of poorly watersoluble drugs. J Bionanosci 2012;2:227-50.

36. Leonardi A, Greco AS, Pignatello R, Fuochi V, Petronio Petronio G Furneri PM. A method for efficient loading of ciprofloxacin hydrochloride in cationic solid lipid nanoparticles: formulation and microbiological evaluation. Nanomaterials 2018;8:2079-4991.

37. Khatak S, Dureja H. Recent techniques and patents on solid lipid nanoparticles as novel carrier for drug delivery. Recent Pat Nanotechnol 2015;9:150-77.

38. Yadav P, Soni G, Mahor A, Alok S, Singh P, Verma A. Solid lipid nanoparticles: an effective and promising drug delivery system-a review. Int J Pharm Sci Res 2014;5:1152.

39. Chattopadhyay P, Shekunov Y, Yim D, Cipolla D, Boyd B, Farr S. Production of solid lipid nanoparticle suspensions using supercritical fluid extraction of emulsions (SFEE) for pulmonary delivery using the AERx system. Adv Drug Delivery Rev 2007;59:444-53.

40. Couto R, Alvarez V, Temelli F. Encapsulation of vitamin B2 in solid lipid nanoparticles using supercritical CO2. J Supercrit Fluids 2017;120:432-42.

41. Singh R. Preparation of solid lipid nanoparticles through various methods using different precursors. J Drug Delivery Ther 2019;9:415-9.

42. Kakadia G, Conway R. Solid lipid nanoparticles: a potential approach for dermal drug delivery. Am J Pharmacol Sci 2014;2:5A.

43. Schwarz Luo Y, Chen D, Ren L, Zhao X, Qin J. Solid lipid nanoparticles for enhancing vinpocetine's oral bioavailability. J Controlled Release 2006;114:53-9.

44. Luo Y, Chen D, Ren L, Zhao X, Qin J. Solid lipid nanoparticles for enhancing vinpocetine's oral bioavailability.J Controlled Release 2006;114:53-9.

45. Butani D, Yewale C, Misra A. Topical amphotericin b solid lipid nanoparticles: design and development. Colloids Surf B 2016;139:17-24.

46. Kunasekaran V, Krishnamoorthy K. Formulation and evaluation of nanoscale solid lipid particles containing a hydrophilic drugrasagiline mesylate. J Appl Pharm Sci 2016;6:44-50.

47. De Assis N, Mosqueira F, Vilela C, Andrade S, Cardoso N. Release profiles and morphological characterization by atomic force microscopy and photon correlation spectroscopy of 99mTechnetium-fluconazole nanocapsules. Int J Pharm 2008;349:152-60.

48. Pal L, Jana U, Manna K, Mohanta P, Manavalan R. Nanoparticle: an overview of preparation and characterization. J Appl Pharm Sci 2011;1:228-34. 
49. Kaliamurthi S, Selvaraj G. Insight on solid lipid nanoparticles: characterization and application in diabetes mellitus. J Crit Rev 2003;3:11-6.

50. Ansari MJ, Anwer MK, Jamil S, Al-Shdefat R, Ali BE, Ahmad MM, Ansari MN. Enhanced oral bioavailability of insulin-loaded solid lipid nanoparticles: pharmacokinetic bioavailability of insulinloaded solid lipid nanoparticles in diabetic rats. Drug Delivery 2016;23:1972-9.

51. Mahajan A, Kaur S, Kaur S. Design, formulation, and characterization of stearic acid-based solid lipid nanoparticles of candesartan cilexetil to augment its oral bioavailability. Asian J Pharm Clin Res 2018;11:344-50.

52. Chetoni P, Burgalassi S, Monti D, Tampucci S, Tullio V, Cuffini $\mathrm{AM}$, et al. Solid lipid nanoparticles as a promising tool for intraocular tobramycin delivery: pharmacokinetic studies on rabbits. Eur J Pharm Biopharm 2016;109:214-23.

53. Bhaskar K, Anbu J, Ravichandiran V, Venkateswarlu V, Rao M. Lipid nanoparticles for transdermal delivery of flurbiprofen: formulation, in vitro, ex vivo and in vivo studies. Lipids Health Disease 2009;8:6.

54. Gonullu U, Uner M, Yener G, Fatma Karaman EC, Aydogmus Z. Formulation and characterization of solid lipid nanoparticles, nanostructured lipid carriers and nanoemulsion of lornoxicam for transdermal delivery. Acta Pharm 2015;65:1-3.

55. Jain S, Jain S, Khare P, Gulbake A, Bansal D, Jain SK. Design and development of solid lipid nanoparticles for topical delivery of an anti-fungal agent. Drug Delivery 2010;17:443-51.

56. Hamsika M, Gowda DV, Vindru J, Moin A. Nanotechnology for ophthalmic preparations. Int J Curr Pharm Res 2016;8:5-11.

57. Rosiere R, Van Woensel M, Gelbcke M, Mathieu V, Hecq J, Mathivet $\mathrm{T}$, et al. New folate-grafted chitosan derivative to improve the delivery of paclitaxel-loaded solid lipid nanoparticles for lung tumor therapy by inhalation. Mol Pharm 2018;15:899-910.
58. Tupal A, Sabzichi M, Ramezani F, Kouhsoltani M, Hamishehkar H. Dermal delivery of doxorubicin-loaded solid lipid nanoparticles for the treatment of skin cancer. J Microencapsulation 2016;33:372-80.

59. Liu J, Hu W, Chen $\mathrm{H}, \mathrm{Ni} \mathrm{Q}, \mathrm{Xu} \mathrm{H}$, Yang X. Isotretinoin-loaded solid lipid nanoparticles with skin targeting for topical delivery. Int J Pharm 2007;328:191-95.

60. Wang W, Zhang L, Chen T, Guo W, Bao X, Wang D, Ren B, et al. Anticancer effects of resveratrol-loaded solid lipid nanoparticles on human breast cancer cells. Molecules 2017;22:1814.

61. Jain K, Chourasia K, Masuriha R, Soni V, Jain A, Jain K, et al. Solid lipid nanoparticles were bearing flurbiprofen for transdermal delivery. Drug Delivery 2005;12:207-15.

62. Bhandari R, Kaur IP. Pharmacokinetics, tissue distribution and relative bioavailability of isoniazid-solid lipid nanoparticles. Int J Pharm 2013;441:202-12.

63. Sarangi MK, Padhi S. Solid lipid nanoparticles-a review. J Critical Rev 2016;3:5-12.

64. Abdelgawad, Nas M, Hamza MY, Awad GAS. Topical and systemic dermal carriers for psoriasis. Int J Curr Pharm Res 2016;8:4-9.

65. Kelidari HR, Saeedi M, Akbari J, Morteza Semnani K, Gill P, Valizadeh H, Nokhodchi A. Formulation optimization and in vitro skin penetration of spironolactone loaded solid lipid nanoparticles. Colloids Surf B 2015;128:473-9.

66. Sailaja K, Amareshwar P, Chakravarty P. Formulation of solid lipid nanoparticles and their applications. Curr Pharma Res 2011;1:197.

67. Souto B, Müller H. Cosmetic features and applications of lipid nanoparticles (SLN@, NLC®). Int J Cosmet Sci 2008;30:157-65.

68. Wissing SA, Muller RH. Cosmetic applications for solid lipid nanoparticles (SLN). Int J Pharm 2003;254:65-8. 